\title{
Digital Templating and Preoperative Deformity Analysis with Standard Imaging Software
}

\author{
Amir A. Jamali MD
}

Received: 5 September 2008 / Accepted: 9 April 2009/Published online: 15 May 2009

(C) The Author(s) 2009. This article is published with open access at Springerlink.com

\begin{abstract}
Analysis of deformity and subsequent correction are the basis for many orthopaedic surgical procedures. In advanced cases of joint degeneration, arthroplasty may be the only available treatment option. Until recently, these analyses and preoperative surgical plans have been performed using standard radiographs, tracing paper, and/or plastic overlays. Numerous customized, commercially available, computer-based preoperative planning software programs have been introduced. The purposes of this study were to describe (1) the techniques used in deformity analysis and preoperative surgical planning using standard radiographs for joint arthroplasty and corrective osteotomies of the extremities, (2) the use of computed tomography (CT) scans to analyze rotational deformities in the presence and absence of joint prostheses and in planning corrective rotational osteotomies or revision joint replacement, and (3) the techniques for analyzing angular deformities of the spine. All these applications were performed with a widely available image analysis software.
\end{abstract}

\section{Introduction}

Precise preoperative planning is a vital principle of success in orthopaedic surgery. Arguably, no field in medicine is as dependent for its success on accurate planning and

The author certifies that he has no commercial associations (eg, consultancies, stock ownership, equity interest, patent/licensing arrangements, etc) that might pose a conflict of interest in connection with the submitted article.

A. A. Jamali $(\bowtie)$

Department of Orthopaedic Surgery, UC Davis Medical Center, 4860 Y Street, \# 3800, Sacramento, CA 95817, USA

e-mail: amir.jamali@ucdmc.ucdavis.edu implementation of alignment correction and implant placement. Traditionally, preoperative planning has been performed on standard radiographs with various techniques, including the use of clear plastic templates [7-9, 11]. Recently, digital templating was proposed as a method to electronically overlay templates from a digital library on clinical radiographs for arthroplasties [3]. The advocates of this technique cite the wide variety of available templates, the speed and precision of the technique, and elimination of hard-copy printouts of radiographs with their associated cost. The disadvantages of digital templating are the dependence on the digital library, cost of the software, and limitations in software design for each application.

The purposes of this study are to describe (1) the techniques used in deformity analysis and preoperative surgical planning using standard radiographs for joint arthroplasty and corrective osteotomies of the extremities, (2) the use of CT scans to analyze rotational deformities in the presence and absence of joint prostheses and in planning corrective rotational osteotomies or revision joint replacement, and (3) the techniques for analyzing angular deformities of the spine. For all these applications, the specific use of a widely available image analysis software is discussed.

\section{Materials and Methods}

For this study I proposed using commercially available software (Adobe ${ }^{\circledR}$ Photoshop ${ }^{\circledR}$ 6.0; Adobe Systems Inc, San Jose, CA) [1] for a wide variety of orthopaedic surgical applications. However, any software program that allows calibration of measurements for lines and angles can be used. These programs are widely available for photographic editing and management. They can be used with a step-by-step technique for orthopaedic applications with 
lower cost and increased flexibility than commercial orthopaedic software (Tables 1-3; Figs. 1, 2).

The alignment objective in TKA is to restore a projected anteroposterior weightbearing axis of the lower extremity to pass through the center of the knee [14]. Traditionally, in the coronal plane, the goal is to perform the distal femoral cut exactly perpendicular to a line from the femoral head to the apex of the femoral notch distally. This axis is defined as the mechanical axis of the femur. The anatomic axis is defined as the line of best fit in the femoral diaphysis that passes through the center of the distal femur. The position of this axis is obtained intraoperatively by placement of an intramedullary rod in the femur starting at a point just anterior to the origin of the posterior cruciate ligament on the inferior trochlea. On the femur, the angle between the mechanical axis and the anatomic axis (Fig. 3A-B) is determined preoperatively. This angle is measured in the software package and usually is between $4^{\circ}$ and $7^{\circ}$ (Fig. 3B, black arrow). This angle is equivalent to the valgus angle set on the distal femoral cutting guide, thus achieving a distal femoral cut perpendicular to the mechanical axis of the femur. On the tibia, the goal is to cut the tibial surface exactly perpendicular to the line connecting the midpoint of the medial and lateral tibial spines and the center of the ankle. This axis is defined as the mechanical axis of the tibia. A perpendicular line is drawn to this mechanical axis line in the software package, which then can be translated proximally and distally depending on the desired degree of bone resection. By placing the distal femoral and proximal tibial cut lines on their respective bones, the profile and thickness of each cut can be predicted preoperatively (Fig. 3B) and both bone cuts will be perpendicular to their respective mechanical axes in the coronal plane. The same strategy can be applied in the sagittal plane with the measurement of the native proximal tibial slope and distal femoral flexion/extension as well as the analysis of any post-traumatic or congenital deformities involving the femur or tibia.

A second objective in planning TKA using digital templating is to select the size of the implants preoperatively. This can be performed by scanning each of the templates into a separate image file and calibrating the templates to the calibration markers placed on the radiograph (Table 2). The preoperative plan can be performed by overlaying the template images digitally on the radiographs (Fig. 3C). This technique allows a close approximation of the ultimate implant sizes preoperatively (Fig. 3D-E).

The goal of THA is to replace the painful hip. For longterm success, many believe that the restoration of hip biomechanics may lead to improved outcomes. With appropriate preoperative planning, the hip center of rotation and the offset between the proximal femur and the acetabulum can be improved. Perhaps more importantly to patients, leg length discrepancies may be corrected. The offset is defined as the lateral displacement of the femur relative to the pelvis. The surgeon can adjust these factors based on the depth of reaming and subsequent cup placement, vertical placement of the femoral implant, type of implant used (standard versus high offset), use of offset polyethylene liners, and variations in femoral head sizes.

Table 1. General techniques for Adobe ${ }^{\circledR}$ Photoshop ${ }^{\circledR}$

Techniques/Steps

1. Placement of lines

A. Multiple layers can be created by going to the layer menu at the top and selecting "New" and then "Layer"

B. Select the "line tool" (Fig. 1, large white arrow) on the "toolbar"

C. Draw the path for the lines by pressing the left mouse button and dragging the mouse to the end of the line and then releasing the left mouse button

D. Finalize the line on the new layer by placing the cursor over the line, pressing the right mouse button and selecting "fill path". The line will now be placed definitively on the new layer established as shown in $1 \mathrm{~A}$

2. Angular measurements

A. Angles can be measured by selecting the "measure tool" (Fig. 1, small black arrows)

B. To measure an Angle ABC, press the left mouse button and drag from Points A to B

C. At this point, a linear measurement is given at the top of the screen

D. Keep the cursor over Point B and press the "Alt" button; the ruler is then converted to an angle icon on the image

E. Press the left mouse button again and drag the mouse to Point $\mathrm{C}$

F. At this point, an angular measurement is given for the Angle ABC in the "Info" window (under "Window" menu at top of screen)

3. Placement of text

A. Creating a new layer and then selecting the "text" tool (Fig. 1, letter T)

B. Press the left click button and drag the mouse to create a text box; text can be typed into the text box

C. If text is not visible, be sure the font is large enough to match the size of the image and change the color to improve contrast 
Table 2. Technique for radiographic templating using Adobe ${ }^{\mathbb{R}}$ Photoshop $^{\mathbb{R}}$

Procedures/Steps

1. Image and template software entry

A. Scan template overlays for desired digital templating using a standard tabletop scanner

B. Convert images to black and white by selecting the "Image" toolbar followed by "Mode"; select "Grayscale"

C. Save template image files in separate folders as jpeg (.jpg) files (select "File" toolbar followed by "Save as" and select a file name with a "Format" as "JPEG")

D. Enter desired radiographs, CT images, or MR images into software by directly downloading them or by scanning them

E. In cases of standard radiographs, some form of calibration template must be placed on the skin at the level of the joint

F. In cases of CT or MRI, the calibration bar from the scanner must be included on the image

G. Select the "Window" menu on the Photoshop menu window at the top of the screen containing the items: "File," "Edit," "Image," "Layer," etc

H. Be sure both "show tools" and "show layers" are selected

I. Collect and save all images used for that specific case on the computer desktop or in a separate folder

$\mathrm{J}$. The preoperative radiograph is opened as the initial image

2. Placement of template image on preoperative radiograph

A. The desired template image is opened in the software

B. The template image is inverted to make it more visible by selecting the "Image" menu, followed by "Adjust," and "Invert" or by pressing Ctrl I

C. From the Photoshop toolbar (Fig. 1), select the top left "Marquee Tool" (black arrow) in the shape of a rectangle

D. On the template image, the "Marquee Tool" is used to outline the template along with a 10-cm segment of the calibration ruler found on most implant templates

E. Absence of a ruler on the template makes size calibration impossible

F. Press Ctrl C to copy this portion of the template image

G. Select the preoperative radiograph image and press Ctrl V to paste the desired portion of the template image onto the preoperative radiograph image

H. At this point, under the "Layers Palette" (Fig. 2), one will see a new layer (that of the template image)

I. Toggle the visibility of this layer by pressing the "visibility toggle," which appears as an eye on the Layers Palette (Fig. 2, black arrow)

J. Change the opacity of the new template image by selecting the template layer on the Layers Palette (Fig. 2) and using the Opacity tool on the upper right of the Layers Palette (Fig. 2, large black arrowhead); slide it to 50\%; at this point, the template image becomes partially transparent

$\mathrm{K}$. Change the brightness/contrast of the new template image by selecting the template layer as described previously and selecting the "Image" toolbar followed by "Adjust"; select "Brightness/Contrast"

3. Transforming the template image

A. Next, the size of the selected portion of the template image is adjusted to the radiograph

B. Press Ctrl T to "free transform" the template layer

C. Once this has been selected, a rectangle encloses the entire template image layer

D. The rectangle can be resized either vertically or horizontally by passing the cursor over any of its sides, pressing the left mouse button, and dragging the mouse

E. The rectangle can be rotated by passing the cursor over its corners, pressing the left mouse button, and dragging the mouse

F. Ensure the magnification change seen at the top of the page is equal for both the height and width to avoid template image distortion

G. Press the Enter button once you have positioned and resized the template image appropriately

H. Repeat the "free transform" process as necessary

4. Repositioning the template image

A. Select the layer of the template image in the Layers Palette (Fig. 2, template image not shown)

B. Use the Move tool in the top right of the Photoshop toolbar (Fig. 1, large black arrowhead)

C. Left click on the template image and drag it to the desired position with the mouse

5. Calibrating the template image

A. Rotate the template image, resize, and reposition it as directed in 3A-E so that its ruler portion superimposes the calibration of the preoperative radiograph; for example, the $100-\mathrm{mm}$ ruler should be exactly matched to the 100 -mm radiographic marker on the skin

B. Once the sizing has been selected, the template image can again be rotated and repositioned (but not resized) and placed in the desired position over the bone 
Table 2. continued

Procedures/Steps

C. The same technique can be performed for multiple templates (ie, femoral and acetabular for THA, femoral and tibial for TKA, calibrated rulers, etc)

D. While working on a new template, make the previous template layers invisible by toggling the layer visibility icon on the Layers Palette (Fig. 2, black arrow)

Table 3. Technique for rotational analysis of CT/MR images using Adobe ${ }^{\circledR}$ Photoshop ${ }^{\circledR}$

Steps

A. Copy and paste desired cuts as separate layers into new image document (Fig. 2)

B. Make all images invisible by toggling the layer visibility icon on the Layers Palette (Fig. 2, black arrow)

C. Alter the opacity of the image listed higher on the layer list to $50 \%$ (Fig. 2, black arrowhead)

D. The images will be superimposed at this point

E. Draw lines along the axes that are to be measured (eg, femoral neck axis, transmalleolar axis, etc) as directed in general techniques (Table 1) F. Measure angles as directed in general techniques (Table 1)

Fig. 1 The toolbar from the software package is shown with common tools needed for digital templating: line tool (white arrow); measure tool (small black arrows); text tool (letter T); marquee tool (black arrow); and move tool (large black arrowhead).

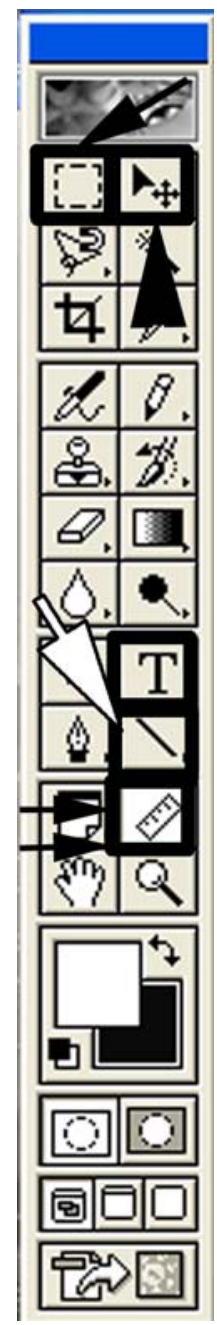

Digital templating allows the surgeon to precisely preplan these factors to restore hip biomechanics and to minimize leg length inequality (Fig. 4).

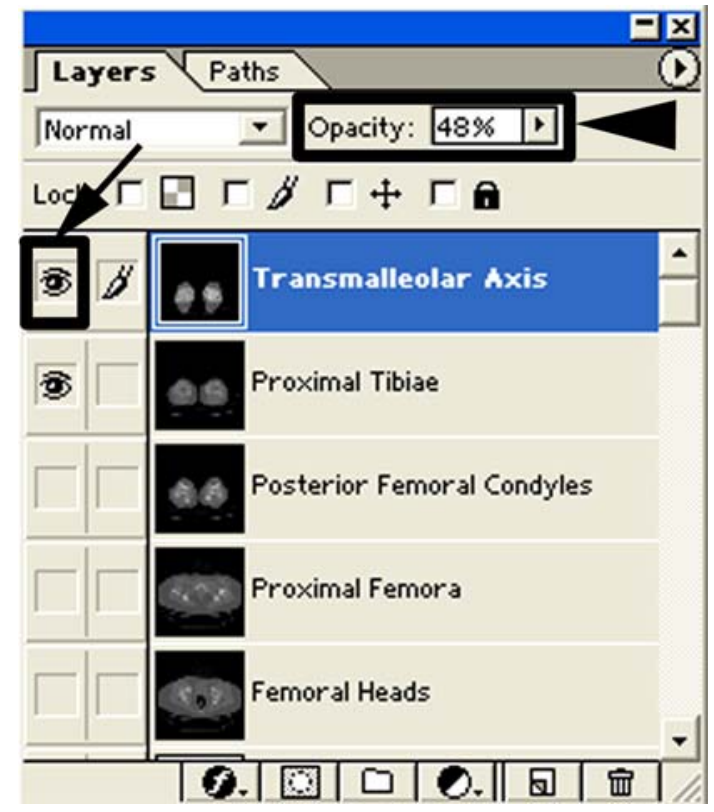

Fig. 2 The software layers palette is shown with visibility toggle (black arrow) and layer opacity adjustment (large black arrowhead).

Resurfacing THA has gained worldwide interest in recent years as a method to address younger patients with advanced hip arthritis while preserving bone for future surgical procedures. The major source of failure for this procedure is on the femoral side. Varus positioning of the femoral component in the coronal plane is one risk factor for such failure resulting from the tensile stresses applied to the lateral neck bone [16]. Notching of the bone of the femoral neck is an additional risk factor [12]. This can be the result of an excessively small femoral component diameter or a femoral preparation that is excessively in the valgus position in which the cylindrical reamer penetrates 
Fig. 3A-E Digital templating of TKA is performed. (A) A preoperative radiograph shows the mechanical axis of the femur connecting the central distal femur and femoral head and the anatomic axis of the femur (the line running from the distal femur center up the femoral shaft). The tibial mechanical axis is shown as the line connecting the midpoint between the medial and lateral tibial eminence and the center of the ankle. (B) A higher-magnification view of lower extremity alignment is shown at the level of the knee. The black arrow represents the angle between the mechanical and anatomic axes of the femur, which can be used to set the distal femoral valgus cut angle used intraoperatively during TKA. (C) The radiographic templates are imported into the software and are pasted onto the preoperative radiograph (these templates do not contain a calibration ruler). Postoperative (D) anteroposterior and (E) lateral radiographs after the TKA are shown.

Fig. 4A-B Digital templating of a standard uncemented THA is performed. (A) A preoperative radiograph with acetabular and femoral components is shown with the implants in the desired positions and with the templates resized to match the image calibration. (B) The postoperative radiograph is shown with final implants.
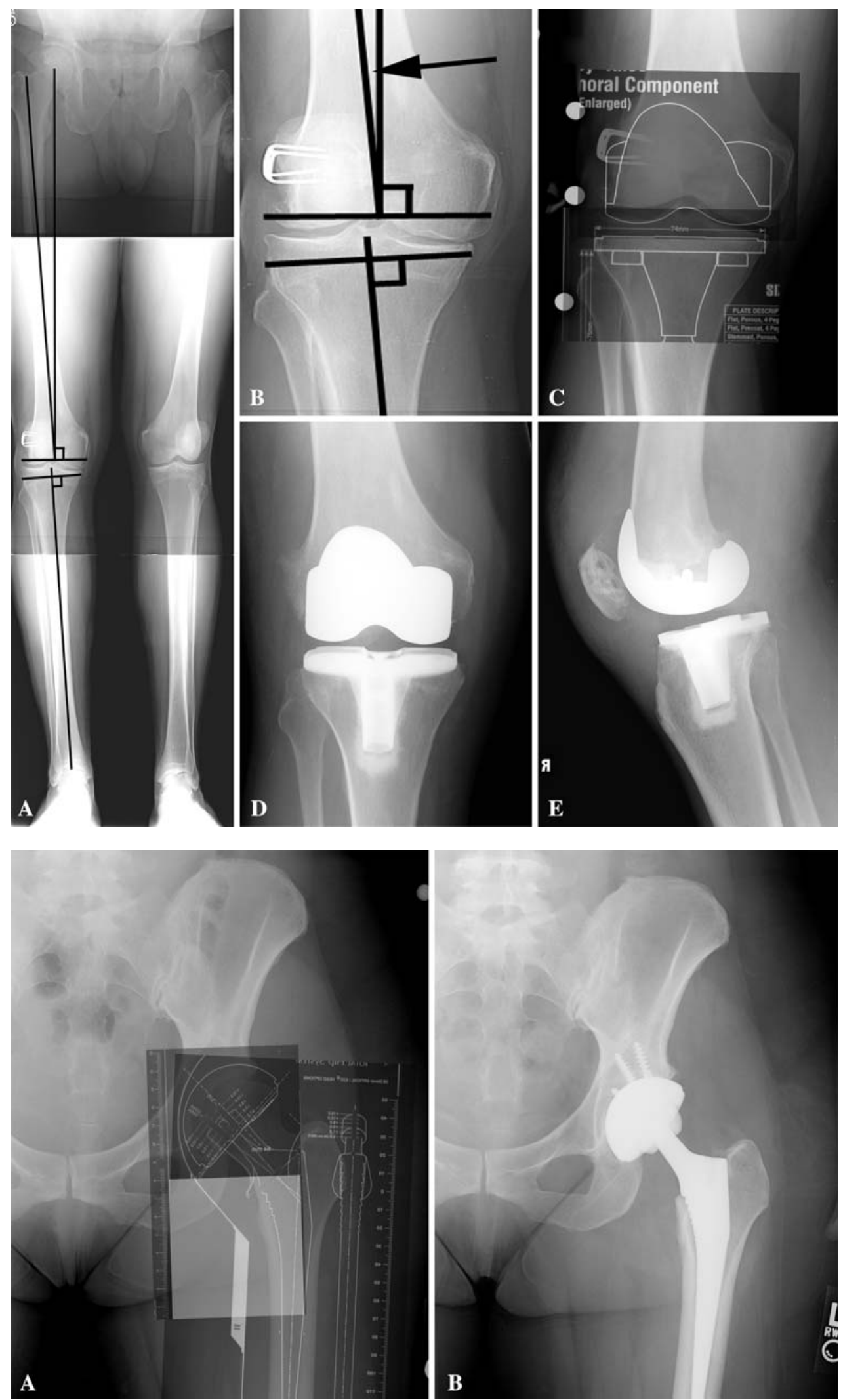

the cortical bone of the lateral neck. On the lateral projection, the femoral head sometimes can be translated posteriorly on the neck forcing the starting point for the guide pin into a more anterior position on the head to remain central in the neck. Additionally, excessive anteversion, retroversion, anterior translation, or posterior 

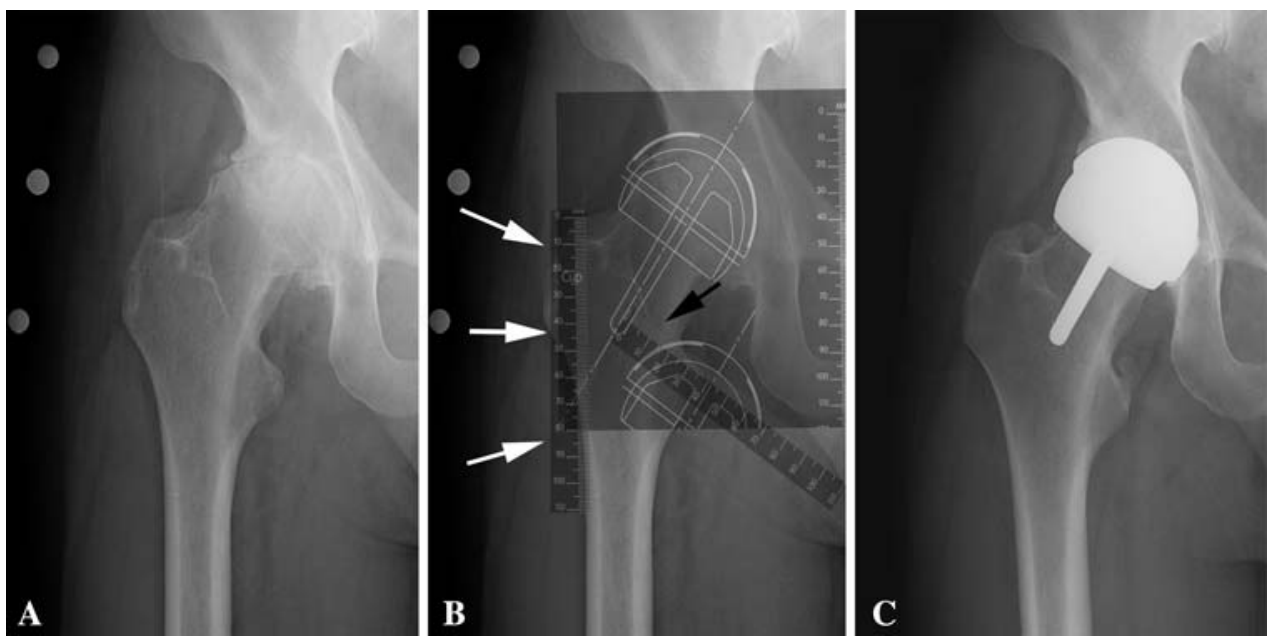

Fig. 5A-C Digital templating of resurfacing THA is performed. (A) A preoperative radiograph shows the size template $(10 \mathrm{~cm})$ on skin. (B) A preoperative radiograph with the calibrated template is shown in the desired position on the femoral component to avoid notching and to achieve the desired stem shaft angle (acetabular sizing requires

translation of the femoral component could lead to notching or impingement against the acetabular component [17]. My preferred technique is to minimize the size of the femoral component while avoiding notching and thus to decrease bone resection on the acetabular side (Fig. 5) [18]. Based on these observations, it is clear there is little room for error in placement of the femoral component in either varus or valgus directions. Some guidance systems use a pin in the lateral femoral cortex to determine the trajectory of the guide pin, measuring the vertical distance from the top of the greater trochanter to the entry site on the femoral shaft $(65 \mathrm{~mm}$; Fig. 5B, white arrows). Others reference the intertrochanteric crest and measure the distance between the projection of the guide pin and the lesser trochanter ( $45 \mathrm{~mm}$; Fig. 5B, black arrow). Both of these measurements can be preplanned by placing calibrated rulers in the desired position in the software.

The indications for osteotomy of the proximal tibia include localized osteoarthritis and deformities of the lower extremity from congenital or traumatic etiologies. I have used the technique of digital templating to preplan a proximal tibial osteotomy for a posttraumatic valgus deformity (Fig. 6) and to perform a virtual correction in the coronal plane with the software. Using this technique, the size and shape of an opening wedge and closing wedge can be predicted and planned before surgery. Additionally, plate templates can be used in the same way as for joint arthroplasty to determine optimal plate length and the potential need for plate bending. The virtually osteotomized tibia can then be freely transformed and rotated until the desired correction is achieved on the image (Fig. 6B). The final mechanical axis of the extremity also can be different positioning of the template). Calibrated rulers have been imported to determine the distance for guide pin placement along the lateral femoral cortex or along the intertrochanteric crest (based on preferred guidance technique). (C) A postoperative anteroposterior hip radiograph is shown.

confirmed on this image to run in the desired position. In addition to the coronal plane planning, the same strategies can be used in correcting congenital or posttraumatic deformities in the sagittal plane and in avoiding an iatrogenic sagittal deformity during correction of a pure coronal plane deformity.

Rotational malalignment of the lower extremity is an important source of disability and pain. In many cases, malalignment can be a source of patellar subluxation and dislocation. Physical examination methods such as the quadriceps angle ( $Q$ angle), J sign, femoral anteversion, and tibial torsion are examiner-dependent and do not provide quantitative information on the degree and location of deformities (ie, femoral or tibial). Thus, axial CT scans of the lower extremity provide a valuable role in measurement of these deformities. By superimposition of these images using the software package and by changing the opacity and visible layers, these relationships can be measured precisely and the optimal corrective procedures can be selected (Table 3). The technique requires a CT scan of the pelvis, which includes the femoral heads down to the lesser trochanters, the knee from the distal femur to the level of the tibial tubercle, and the ankle to include both malleoli. To determine the femoral neck axis, various techniques have been advocated. I have used the method described by Teitge and Torga-Spak [15] (Fig. 7A) by superimposing the axial image with the femoral head center on a second axial image at the base of the neck with its center. Using the layering functions of the software, a line is drawn connecting these two points (Tables 1,2). A second line is drawn along the posterior femoral condyles (Fig. 7B) to determine the axis or rotation of the knee. The angle 

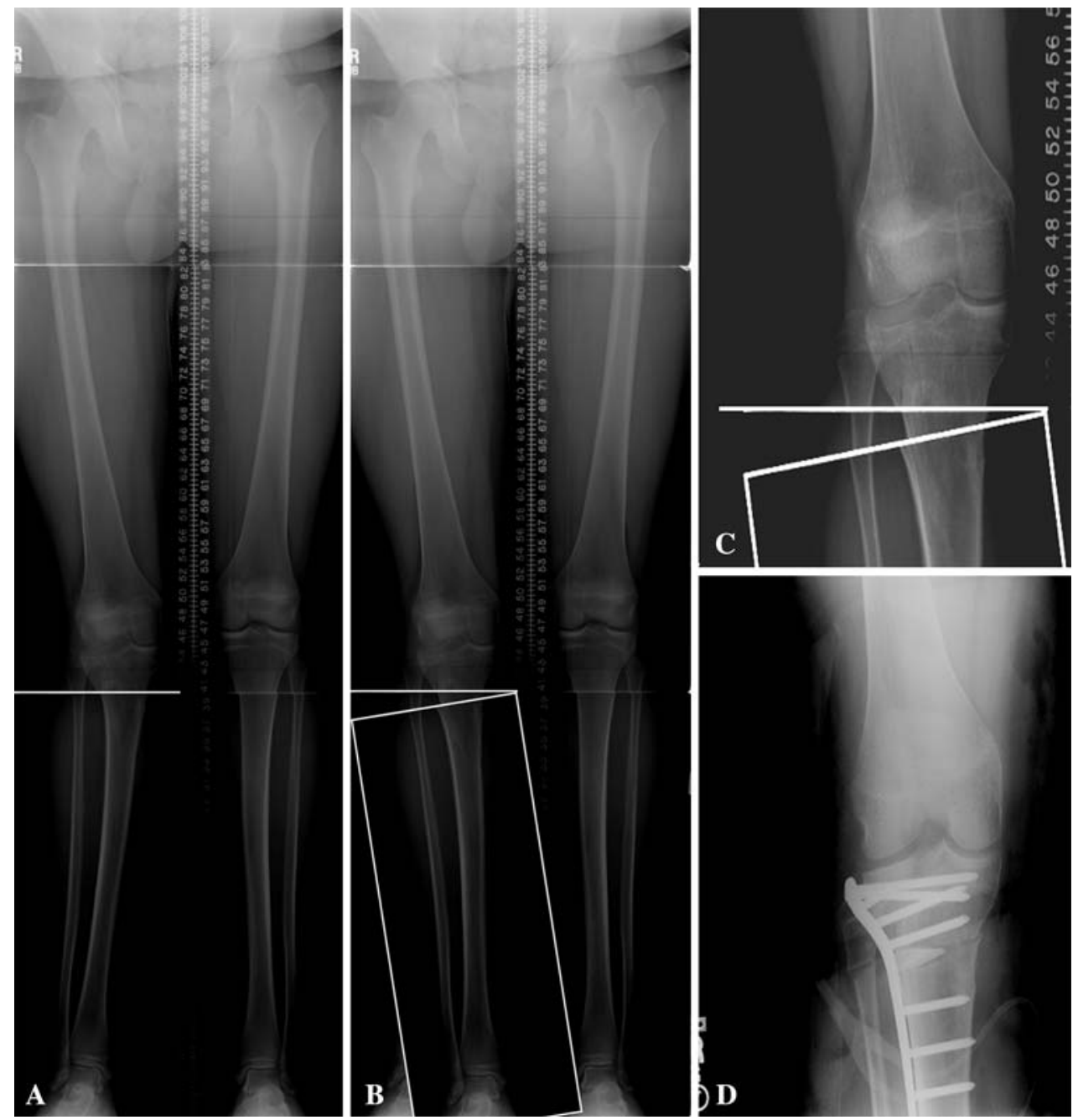

Fig. 6A-D Digital preoperative planning of opening wedge varus high tibial osteotomy in a posttraumatic deformity is shown. In this case, a lateral opening wedge osteotomy is selected. (A) Osteotomy level selection: The level of the initial transverse osteotomy is drawn on the long, standing radiograph. Next, the selection tool is used to select a rectangle to include the entire tibia, ankle, and foot (not shown). (B) Osteotomy fragment reorientation: This image selection is copied and pasted into a new layer in the software. The virtually osteotomized tibia then can be rotated using the "free transform" function (white box). This view allows assessment of desired

between the femoral neck axis and the posterior femoral condylar line is measured and defined as the femoral anteversion. This can be performed by superimposition of the layers of the distal femur and those of the femoral neck and head (Fig. 7C). An identical technique can be used to determine external tibial torsion (Fig. 7D, white arrow) using a coronal line bisecting the tibial plateau. The angle between this line and the transmalleolar axis (Fig. 7D, black arrowhead) defines the external tibial torsion. Measurements of these values are important in the determination of congenital and posttraumatic deformities and in planning corrective osteotomies. correction by drawing a line from the center of the femoral head to the center of the ankle (not shown). The final mechanical axis of the extremity also can be confirmed on this image to run in the desired position and the lateral cortical opening can be measured on the preoperative plan. (C) A high magnification image of B is shown. (D) Postoperative radiograph: A postoperative radiograph was obtained after stabilization with a locking plate (TomoFix ${ }^{\mathrm{TM}}$; Synthes, Paoli, PA) and after placement of a tricalcium phosphate wedge in the osteotomy site.

The analysis of rotation has important implications for evaluating patients with painful TKA. Berger et al. [2] used rotational analysis for assessment of patellar subluxation and dislocation in TKA. They compared 30 patients with patellofemoral complications after TKA with 20 patients with successful outcomes. They developed a method to measure the component rotation of the femoral and tibial components independently by superimposing the axial CT images. Femoral component rotation was measured relative to the transepicondylar axis and tibial component rotation was measured relative to the tibial tubercle (Fig. 8). They reported a combined internal rotation of $1^{\circ}$ to $4^{\circ}$ was 
Fig. 7A-D The rotational alignment can be determined using axial CT images. (A) Superimposed axial images of the femoral head centers and bases of femoral necks are shown. The black lines delineate the femoral neck axis. (B) An axial CT scan of the distal femur is shown with posterior condylar lines. (C) Images $\mathrm{A}$ and $\mathrm{B}$ are superimposed. The angle between the posterior condylar lines and femoral neck axis is defined as the femoral anteversion. (D) Tibial torsion is measured by bisecting the proximal tibiae (white arrow) and defining the transmalleolar axis (black arrowhead). The angle between the two lines is defined as the tibial torsion.
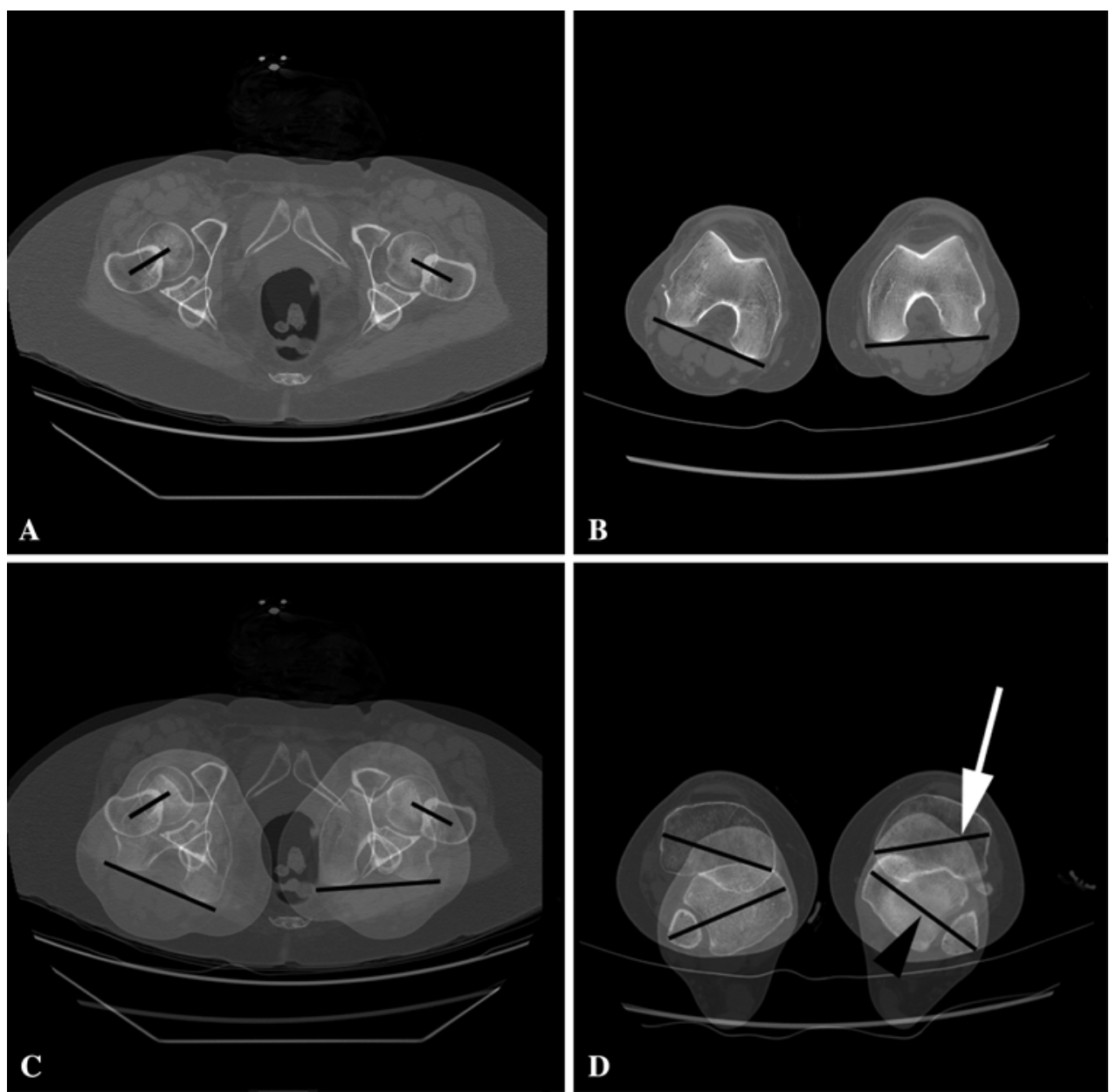

associated with lateral tracking and patellar tilt. A combined internal rotation of $3^{\circ}$ to $8^{\circ}$ was associated with patellar subluxation. Larger degrees of combined internal rotation $\left(7^{\circ}-17^{\circ}\right)$ were associated with early patellar dislocation or late patellar failure. In the case of TKA, the CT images need only include the knee down to the level of the tibial tubercle unless there is an issue regarding proximal femoral or distal tibial deformity. Using this technique, the relationship between the transepicondylar axis (Fig. 8A, black arrow) and the prosthetic posterior femoral condylar axis can be determined (Fig. 8A, white arrow). Additionally, the relationship between the tibial component (Fig. 8B) and the tibial tubercle (Fig. 8C) can be evaluated to determine whether tibial malrotation is an underlying factor leading to patellar subluxation or dislocation (Fig. 8D).

Numerous methods have been used to measure kyphotic, scoliotic, and pelvic deformities in the axial skeleton. They can include measurements of the Cobb angle [6] in scoliosis, kyphotic angles after vertebral fractures, and pelvic obliquity. The techniques described in this article can be used in making these assessments. One such example would be measurement of the kyphotic angle in guiding operative versus nonoperative treatment of burst fractures of the spine (Fig. 9). In the method described by Knight et al. [10], the angle subtended by a line overlying the inferior end plate of the vertebra above the fracture and the line overlying the inferior end plate of the fractured vertebra is measured using the techniques described here (Fig. 9C).

\section{Discussion}

The purpose of this article is to provide technical guidelines for use of a widely digital imaging software program to achieve three objectives: the use of deformity analysis and preoperative surgical planning of standard radiographs in joint arthroplasty and corrective osteotomies, the use of CT scans to analyze rotational deformities, and the analysis of angular deformities of the spine. Using these techniques, preoperative planning can be performed from anywhere and at any time as long as the images can be scanned or downloaded directly from the radiographic archive into the 
Fig. 8A-D Rotational malalignment in TKA can be determined according to the method of Berger et al. [2]. (A) The transepicondylar axis (black arrow) and posterior condylar axis of the prosthesis (white arrow) are marked on this axial CT section of the distal femur. (B) An axial CT scan of the proximal tibia is shown at the level of the polyethylene liner with the center point of the liner shown (white dot). (C) An axial $\mathrm{CT}$ of the proximal tibia is shown at the level of the tibial tubercle. (D) Images B and C are superimposed. The tibial component rotation as defined by Berger et al. [2] is calculated as the angle between a line bisectanterior to posterior running through the center point and a and running through the center point. ing the insert sagittally from line bisecting the tibial tubercle
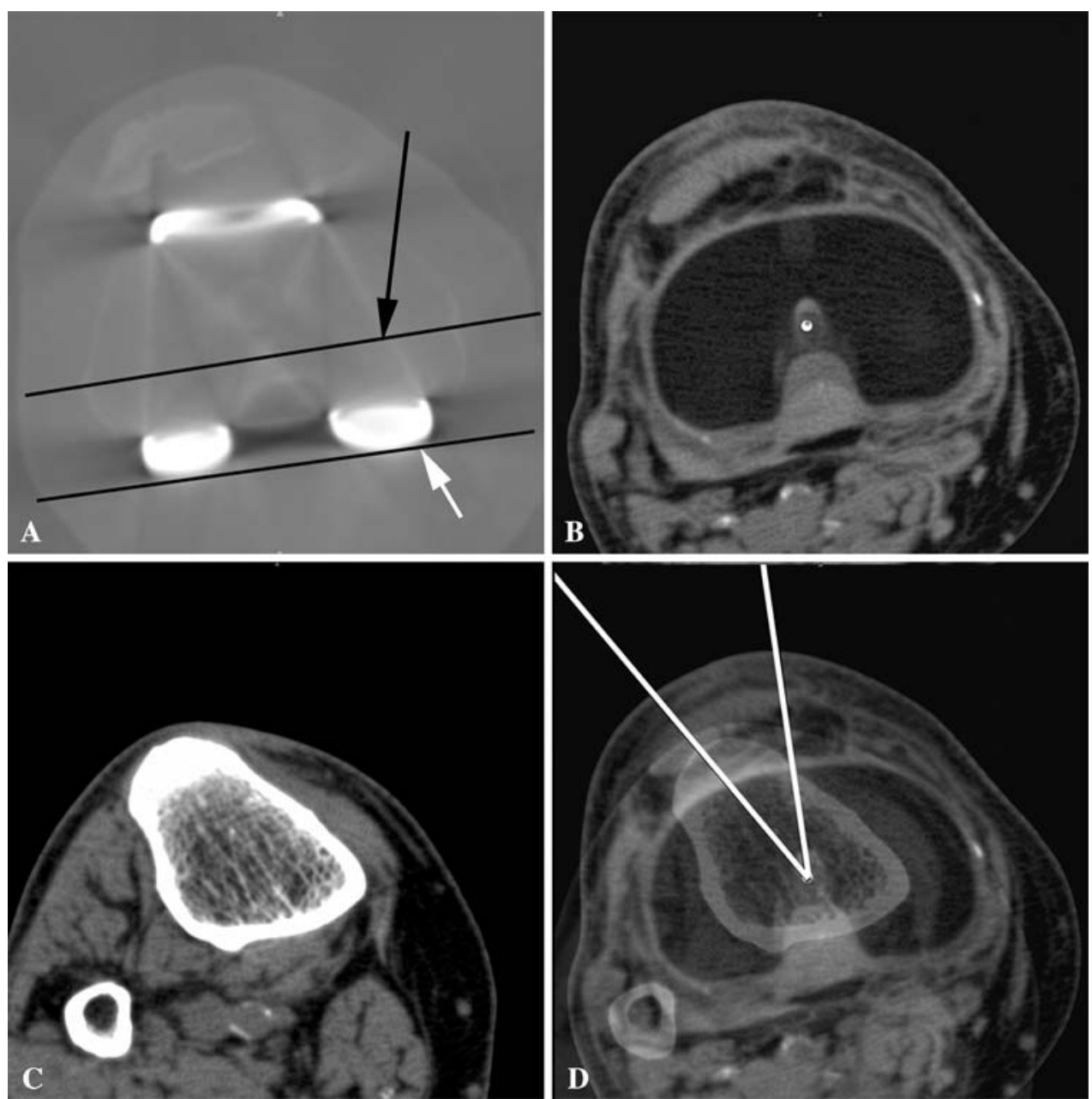

Fig. 9A-C The kyphotic angle for a thoracic burst fracture is calculated according to the method of Knight et al. [10]. (A) A lateral radiograph of the thoracic spine is shown from a patient who has sustained a burst fracture. (B) The fractured vertebral body is outlined. (C) The kyphotic angle is measured according to the method of Knight et al. [10].
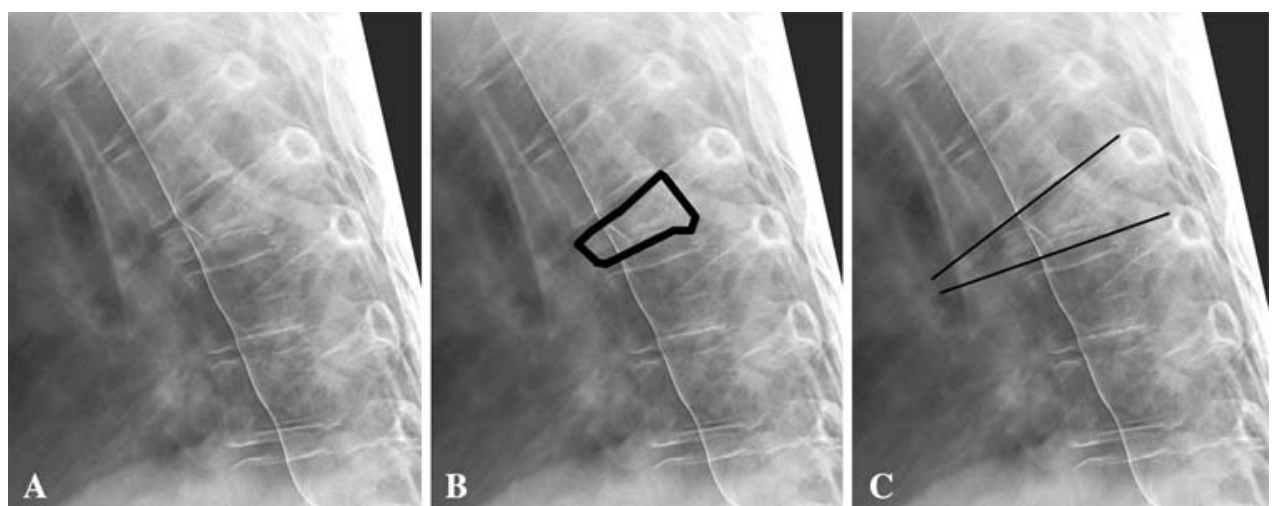

software program. The critical requirements of any software program used for this application include the ability to select and resize certain segments of any image, the use of multiple layers, and the ability to alter the opacity of various layers. The same approaches can be used for essentially any orthopaedic procedure requiring linear or angular correction and/or implantation of size-specific implants.

The approach suggested has some important limitations. The techniques are limited to two-dimensional imaging and each view needs to be templated independently. This limitation makes management of complex three-dimensional deformities difficult, if not impossible. Another limitation of this technique is that the accuracy and precision have not been validated. The techniques require a baseline investment of time from each operator with a variable learning curve. This can lead to insufficient interobserver and intraobserver reliability. Finally and most importantly, when used in preoperative planning, the techniques presented 
here provide a tool to place the implants and perform osteotomies based on a preoperative goal. However, the ideal position of implants and extremity alignment in arthroplasty and corrective osteotomies are points of ongoing debate.

Adobe ${ }^{\mathbb{R}}$ Photoshop ${ }^{\mathbb{R}}$ has been used in some medical and dental applications. Chalazonitis et al. [5] detailed a method to optimize images of radiographs obtained with a digital camera using Photoshop ${ }^{\mathbb{R}}$ software. They described several of the techniques discussed in this article. However, the emphasis of their study was not on preoperative planning and/or the use of the overlay template technique discussed in this article. The segmentation of MR images has been a difficult challenge often requiring either semiautomated or fully manual segmentation of images to separate bone from the surrounding soft tissues. Park et al. [13] described the use of special functions of Photoshop ${ }^{\circledR}$ software to perform semiautomatic segmentation of MR images. They then used these segmented images to make three-dimensional reconstructions of various anatomic structures, including the skin, bones, digestive tract, respiratory tract, urinary tract, cardiovascular system, and nervous system. They were able to fulfill their objective of substantially accelerating the segmentation process over manual segmentation. In yet another medical application of the same type of software, Carvalho et al. [4] described a digital subtraction radiography technique to evaluate chronic periapical dental lesions. The progression of these lesions was followed with serial radiographs. Traditionally, these evaluations have been qualitative. However, by using the digital subtraction technique, the authors were able to determine if the lesions were healing or expanding and also to quantify the size of the osseous lesions at each time point. The techniques for image rotation, sizing, and the use of layers are similar to those discussed in the current article.

Despite the limitations, the techniques presented here recapitulate the same techniques that have been used for decades using planning tools such as tracing paper, plastic templates, and printed radiographs. The ultimate objective of this study was to show that a universally available digital imaging software package can be used for a wide variety of orthopaedic applications, including analysis of deformities, size-specific templating of implant-related procedures, and planning of corrective surgical procedures. With progression of the digital age and gradual elimination of hard-copy radiographs, digital deformity analysis and surgical planning may soon become the standard technique.
Open Access This article is distributed under the terms of the Creative Commons Attribution Noncommercial License which permits any noncommercial use, distribution, and reproduction in any medium, provided the original author(s) and source are credited.

\section{References}

1. Adobe Photoshop 6.0 User Guide. San Jose, CA: Adobe Systems; 2000.

2. Berger RA, Crossett LS, Jacobs JJ, Rubash HE. Malrotation causing patellofemoral complications after total knee arthroplasty. Clin Orthop Relat Res. 1998;356:144-153.

3. Bono JV. Digital templating in total hip arthroplasty. J Bone Joint Surg Am. 2004;86(suppl 2):118-122.

4. Carvalho FB, Goncalves M, Tanomaru-Filho M. Evaluation of chronic periapical lesions by digital subtraction radiography by using Adobe Photoshop CS: a technical report. $J$ Endod. 2007;33:493-497.

5. Chalazonitis AN, Koumarianos D, Tzovara J, Chronopoulos P. How to optimize radiological images captured from digital cameras, using the Adobe Photoshop 6.0 program. J Digit Imaging. 2003;16:216-229.

6. Cobb JR. Outline for the study of scoliosis. Instr Course Lect. 1948;5:261-275.

7. Eggli S, Pisan M, Muller ME. The value of preoperative planning for total hip arthroplasty. J Bone Joint Surg Br. 1998;80:382-390.

8. Heal J, Blewitt N. Kinemax total knee arthroplasty: trial by template. J Arthroplasty. 2002;17:90-94.

9. Knight JL, Atwater RD. Preoperative planning for total hip arthroplasty: quantitating its utility and precision. $J$ Arthroplasty. 1992;7(suppl):403-409.

10. Knight RQ, Stornelli DP, Chan DP, Devanny JR, Jackson KV. Comparison of operative versus nonoperative treatment of lumbar burst fractures. Clin Orthop Relat Res. 1993;293:112-121.

11. Linclau L, Dokter G, Peene P. Radiological aspects in preoperative planning and postoperative assessment of cementless total hip arthroplasty. Acta Orthop Belg. 1993;59:163-167.

12. Marker DR, Seyler TM, Jinnah RH, Delanois RE, Ulrich SD, Mont MA. Femoral neck fractures after metal-on-metal total hip resurfacing: a prospective cohort study. $J$ Arthroplasty. 2007;22(suppl 3):66-71.

13. Park JS, Chung MS, Hwang SB, Lee YS, Har DH. Technical report on semiautomatic segmentation using the Adobe Photoshop. J Digit Imaging. 2005;18:333-343.

14. Sikorski JM. Alignment in total knee replacement. J Bone Joint Surg Br. 2008;90:1121-1127.

15. Teitge RA, Torga-Spak R. Skeletal malalignment and anterior knee pain: rationale, diagnosis, and management. In: Sanchis-Alfonso V, ed. Anterior Knee Pain and Patellar Instability. Ed 1. London, UK: Springer; 2005:185-199.

16. Vail TP, Glisson RR, Dominguez DE, Kitaoka K, Ottaviano D. Position of hip resurfacing component affects strain and resistance to fracture in the femoral neck. J Bone Joint Surg Am. 2008;90:1951-1960.

17. Vendittoli PA, Ganapathi M, Nuno N, Plamondon D, Lavigne M. Factors affecting hip range of motion in surface replacement arthroplasty. Clin Biomech (Bristol, Avon). 2007;22:1004-1012.

18. Vendittoli PA, Lavigne M, Girard J, Roy AG. A randomised study comparing resection of acetabular bone at resurfacing and total hip replacement. J Bone Joint Surg Br. 2006;88:997-1002. 